\title{
铁镍合金纳米颗粒镶嵌的多级孔氮掺杂碳催化剂的制备 及析氧性能研究
}

\author{
王艺霖王敏杰李静* 魏子栋* \\ (重庆大学化学化工学院 重庆 400044)
}

\begin{abstract}
摘要 非贵金属铁镍合金催化剂在析氧反应(OER)中性能优异, 表现出取代贵金属 $\mathrm{RuO}_{2}$ 催化剂的巨大潜力. 以 $\mathrm{SiO}_{2}$ 为 大孔模板, 多巴胺为氮碳源, $\mathrm{Fe}^{3+}, \mathrm{Ni}^{2+}$ 为金属源, 通过原位吸附、聚合、焙烧、刻蚀等步骤制备得到铁镍合金纳米颗粒 镶嵌的多级孔氮掺杂碳催化剂. 碱性介质中的析氧反应测试表明, 合金催化剂达到电流密度 $10 \mathrm{~mA} \cdot \mathrm{cm}^{-2}$ 时过电位仅为 $286 \mathrm{mV}$, 显著低于以 $\mathrm{RuO}_{2}$ 为催化剂的 $380 \mathrm{mV}$ 过电位; 同时经过 2000 圈循环伏安老化后活性几乎无衰减, 稳定性高. 所制备的合金催化剂具有两方面结构优势: (1)铁镍合金以及单质铁纳米颗粒镶嵌于大孔碳的薄层孔壁中，有利于暴露 活性位点; (2)石墨化氮碳层对合金纳米颗粒的保护提高了材料抗腐蚀性，进而提升其稳定性.
\end{abstract}

关键词＼cjkstart多级孔氮掺杂碳; 铁镍合金; 析氧反应

\section{Iron/nickel Alloy Nanoparticles Embedded in N-doped Porous Carbon for Robust Oxygen Evolution Reaction}

\author{
Wang, Yilin Wang, Minjie Li, Jing* Wei, Zidong* \\ (School of Chemistry and Chemical Engineering, Chongqing University, Chongqing 400044)
}

\begin{abstract}
Hydrogen, a clean, efficient and sustainable energy, can be produced via electrochemical water splitting, during which two key processes, hydrogen evolution reaction (HER) and oxygen evolution reaction (OER), occur simultaneously at the two electrodes of an electrolytic cell. Nevertheless compared to the two-electron process of HER, OER, a four-electron process, is of the inherently kinetic hysteresis step, which can dramatically diminish the overall energy conversion efficiency. The highly active noble-metal-based catalyst $\mathrm{RuO}_{2}$ is considered to be one of the most efficient state-of-the-art OER catalysts. However the high cost and element scarcity significantly hinder their practical applications. Thus it is particularly urgent to develop highly-active but low-cost non-noble-metal alternatives. Iron/nickel alloy based catalysts have been widely studied owing to their promising performances. In this work, we prepared iron/nickel alloy nanoparticles embedded in N-doped hierarchically porous carbon $\left(\mathrm{Fe}_{0.64} \mathrm{Ni}_{0.36} @ \mathrm{NC}\right)$, by simultaneously adsorbing metal precursors and dopamine on surface of $\mathrm{SiO}_{2}$ macroporous hard templates, and then annealing the system and etching the templates. In electrochemical measurements, $\mathrm{Fe}_{0.64} \mathrm{Ni}_{0.36} @ \mathrm{NC}$ shows a superior OER activity in alkaline solution, which only needs an overpotential as low as $286 \mathrm{mV}$ to deliver a current density of $10 \mathrm{~mA} \cdot \mathrm{cm}^{-2}$, being significantly lower than the value of $380 \mathrm{mV}$ for $\mathrm{RuO}_{2}$. Besides, the catalyst displays no obvious activity decrease after 2000 cycles of continuous CV scanning, corresponding to an excellent durability. The observed nice performances of the alloy catalyst in alkaline solution can be ascribed to two critical structural features: (1) the macroporous structures made by stacking of $\mathrm{SiO}_{2}$ microspheres own relatively thin layer of carbon framework, thus the embedded iron/nickel alloy particles can well activate the surrounding carbon layer to expose copious active sites; (2) the graphitized N-doped carbon layers well protect the alloy nanoparticles from corrosion, thus improving the durability of the catalysts. This work gave a nice design for the highly efficient non-noble-metal OER catalysts.
\end{abstract}

Keywords N-doped porous carbon; iron/nickel alloys; oxygen evolution reaction

\section{1 引言}

经济和科技的持续发展使能源需求成为人们关注 的热点. 然而近年来传统化石能源大量开采, 储量日渐 减少，需求量却逐年增大，同时传统化石能源燃烧产生 的废气、废渣造成严重的环境污染、温室效应等问题，使
能源危机和环境问题日益加重 ${ }^{[1 ~ 5]}$. 为了解决上述问题, 发展可持续的清洁新能源和不断提高能源转换效率成 为现代社会最迫切的需求 ${ }^{[6,7]}$. 氢能作为一种重要的储 存和转运能源的载体, 具有燃烧热高、能源转换过程无 毒、无污染等优点 ${ }^{[8 \sim 11]}$. 电解水制氢是氢能生产最便捷

*E-mail: lijing@cqu.edu.cn; zdwei@cqu.edu.cn

Received August 30, 2018; published November 27, 2018.

Supporting information for this article is available free of charge via the Internet at http://sioc-journal.cn.

Project supported by the National Key Research and Development Program of China (No. 2016YFB0101202) and the National Natural Science Foundation of China (Nos. 91534205, 21436003).

项目受国家重点研发计划(No. 2016YFB0101202)和国家自然科学基金(Nos. 91534205, 21436003)资助. 
的途径之一, 可分解为析氢(HER)和析氧(OER)两个半 反应, 其中 OER 为四电子转移过程, 动力学迟缓, 过电 位高, 造成整体转换效率不高 ${ }^{[12 \sim 17]}$. 目前广泛采用 $\mathrm{RuO}_{2}$ 和 $\mathrm{IrO}_{2}$ 等贵金属作为 OER 催化剂, 其活性较好, 但成本高、储备稀有、稳定性差, 无法得到广泛应 用 ${ }^{[18 ~ 21]}$. 因此, 开发高效耐用、储量丰富和高性能的替 代催化剂是近年来大量研究工作的重点. 研究表明过渡 金属基材料, 例如 $\mathrm{Fe}, \mathrm{Co}, \mathrm{Ni}, \mathrm{Mn}$ 等的化合物能够更多 暴露 $\mathrm{d}$ 轨道电子 ${ }^{[22 ~ 28]}$, 提高催化活性, 有望成为可替代 贵金属的析氧催化剂.

最近研究发现, $\mathrm{Fe} / \mathrm{Ni}$ 基催化剂对 $\mathrm{OER}$ 具有较高催 化活性 ${ }^{[29]}$. 例如 Landon 等 ${ }^{[30]}$ 合成了 $\mathrm{Fe} / \mathrm{Ni}$ 双层氢氧化物 $(\mathrm{Fe} / \mathrm{Ni}-\mathrm{LDH})$ 催化剂并与多壁碳纳米管进行复合, 通过 调控催化剂的局部电子结构提高 OER 性能 ${ }^{[31 ~ 35]}$. 同时 $\mathrm{Fe} / \mathrm{Ni}$ 基催化剂与导电基底相结合可加速电荷转移, 促 进 OER 活性, 增强其稳定性 ${ }^{[36,37]}$. 例如, Yang 等 ${ }^{[38]}$ 采用 氮掺杂石墨烯将二元合金或三元合金封装后进行退火 处理, 所得的三元 $\mathrm{Fe} / \mathrm{Co} / \mathrm{Ni}$ 合金-石墨烯催化剂具有良 好的 OER 性能, 包裹在金属合金上的薄碳层能够优化 其吸附能力 ${ }^{[39,40]}$. 同时氮原子的掺杂不仅可以改变碳的 电子状态, 调节表面电子结构以降低局部功函数, 还可 以改变反应中间体的吸附强度并提供 $\mathrm{C}-\mathrm{N}$ 活性位 点 ${ }^{[41,42]}$. 此外大量研究表明, $\mathrm{Fe} / \mathrm{Ni}$ 基催化剂与掺杂碳壳 之间存在协同效应，高度分散的铁镍合金颗粒可以提供 更多的活性位点，同时具有 $3 \mathrm{~d}$ 轨道的金属合金可以通过 有效调节电子结构和吸附能达到提升催化活性的目的.

但是, 在电化学循环过程中, 过渡金属催化剂经过 多次循环反应后容易直接暴露于电解质溶液中, 发生溶 解和颗粒团聚的现象, 造成活性快速下降. 此外, 还存 在铁镍合金颗粒被过厚碳层包裹, 不利于活性位点暴露 等问题.

本项工作制备了铁镍合金纳米颗粒镶嵌的多级孔 氮掺杂碳催化剂 $\mathrm{Fe}_{0.64} \mathrm{Ni}_{0.36} @ \mathrm{NC}$. 以 $\mathrm{SiO}_{2}$ 纳米小球为大 孔模板, 多巴胺 $(\mathrm{PDA})$ 为氮碳源, 不同浓度比例的 $\mathrm{Fe}^{3+}$, $\mathrm{Ni}^{2+}$ 为金属源, 通过原位吸附、聚合、焙烧、 $\mathrm{NaOH}$ 刻 蚀等步骤制备得到 $\mathrm{Fe}_{0.64} \mathrm{Ni}_{0.36}$ 嵌入的 $\mathrm{NC}$ 多级孔结构, 合成过程如图 1 所示. 测试结果表明, 将合金颗粒镶嵌 进薄碳涂层中不仅降低了金属物种在反应过程中的溶 解和团聚程度, 明显提高稳定性及电导率, 还克服了铁 镍合金被包裹在过厚碳层中不利于暴露活性位点等问 题.

\section{2 结果与讨论}

\section{1 电极材料的组成与形貌表征}

首先, 对加入不同比例 $\mathrm{Fe}^{3+}, \mathrm{Ni}^{2+}$ 的样品进行了 $\mathrm{X}$ 射线衍射(XRD)测试(图 2). 只加入 $\mathrm{Ni}^{2+}$ 的样品在 $2 \theta=$ $44.5^{\circ}, 51.8^{\circ}$ 和 $76.3^{\circ}$ 出现衍射峰 ${ }^{[43]}$, 分别对应 $\mathrm{Ni}(111)$, (200)和(220)晶面(JCPDS 卡, \#04-0850), 表明金属镍掺

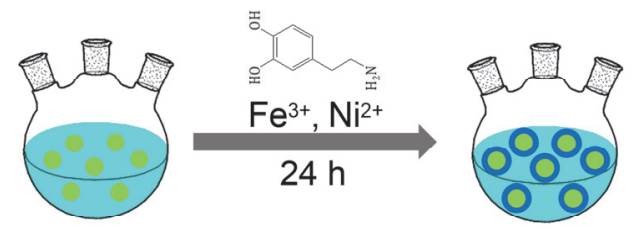

- $\mathrm{SiO}_{2}$

$\mathrm{Fe}^{3+}, \mathrm{Ni}^{2+}-\mathrm{PDA} @ \mathrm{SiO}_{2}$ $800{ }^{\circ} \mathrm{C}$

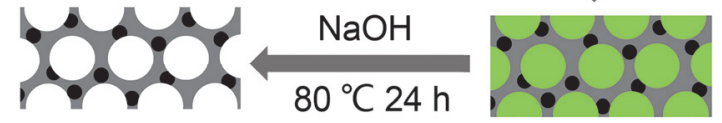

O $\mathrm{Fe}_{0.64} \mathrm{Ni}_{0.36} @ \mathrm{NC}$

- $\mathrm{Fe}_{0.64} \mathrm{Ni}_{0.36} @ \mathrm{NC}-\mathrm{SiO}_{2}$

图 $1 \quad \mathrm{Fe}_{0.64} \mathrm{Ni}_{0.36} @ \mathrm{NC}$ 的合成示意图

Figure 1 The synthetic process for $\mathrm{Fe}_{0.64} \mathrm{Ni}_{0.36} @ \mathrm{NC}$

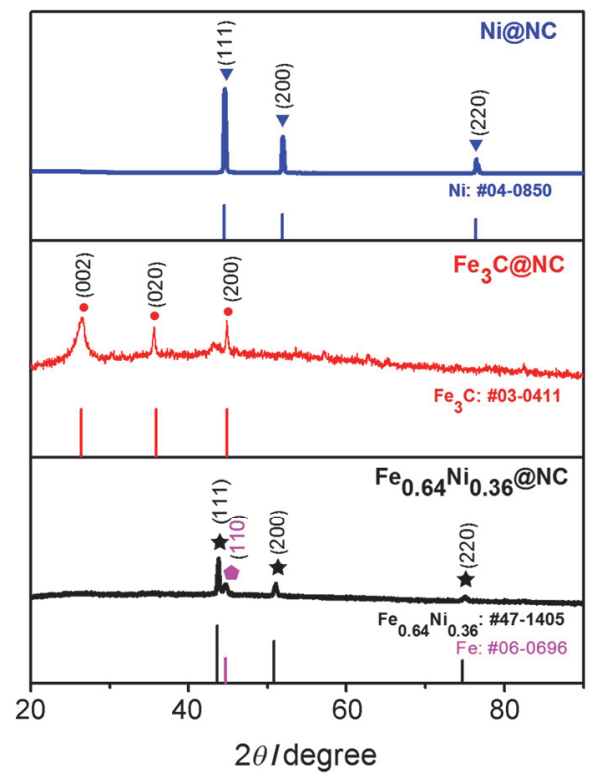

图 $2 \mathrm{Fe}_{0.64} \mathrm{Ni}_{0.36} @ \mathrm{NC}, \mathrm{Fe}_{3} \mathrm{C} @ \mathrm{NC}, \mathrm{Ni} @ \mathrm{NC}$ 的 XRD 图

Figure $2 \mathrm{XRD}$ patterns of $\mathrm{Fe}_{0.64} \mathrm{Ni}_{0.36} @ \mathrm{NC}, \mathrm{Fe}_{3} \mathrm{C} @ \mathrm{NC}$ and $\mathrm{Ni} @ \mathrm{NC}$

杂进入氮碳层, 命名为 $\mathrm{Ni} @ \mathrm{NC}$. 只加入 $\mathrm{Fe}^{3+}$ 的样品在 $2 \theta=26.4^{\circ}, 35.8^{\circ}$ 和 $42.1^{\circ}$ 出现衍射峰, 分别对应 $\mathrm{Fe}_{3} \mathrm{C}(002),(020)$ 和(200)晶面(JCPDS 卡, \#03-0411), 命名 为 $\mathrm{Fe}_{3} \mathrm{C} @ \mathrm{NC}$. 同时加入 $\mathrm{Fe}^{3+}, \mathrm{Ni}^{2+}$ 的样品呈现四个衍射 峰，其中三个 $\left(2 \theta=43.6^{\circ}, 50.8^{\circ}, 74.6^{\circ}\right)$ 可以归属到合金 $\mathrm{Fe}_{0.64} \mathrm{Ni}_{0.36}$ (JCPDS 卡, \#47-1405), 而处在 $2 \theta=44.6^{\circ}$ 的衍 射峰来自于金属 $\mathrm{Fe}(110)$ 晶面衍射(JCPDS 卡, \#06-0696), 因此该样品中金属成分主要为 $\mathrm{Fe}_{0.64} \mathrm{Ni}_{0.36}$ 合金, 同时包 含少量单质铁, 命名为 $\mathrm{Fe}_{0.64} \mathrm{Ni}_{0.36} @ \mathrm{NC}$. 通过电感耦合 等离子体发射光谱法(ICP)测定 $\mathrm{Fe}_{0.64} \mathrm{Ni}_{0.36} @ \mathrm{NC}$ 催化剂 中 $\mathrm{Fe}: \mathrm{Ni}$ 原子比约为 $4: 1$, 表明催化剂中除了含有 $\mathrm{Fe}_{0.64} \mathrm{Ni}_{0.36}$ 合金, 还含有少量 $\mathrm{Fe}$ 单质, 与 $\mathrm{XRD}$ 分析结果 一致. 
利用 $X$ 射线光电子能谱(XPS)方法进一步探究 $\mathrm{Fe}_{0.64} \mathrm{Ni}_{0.36} @ \mathrm{NC}$ 中的元素组成和表面化学态. $\mathrm{N}$ 元素高 分辨图谱(图 3a)可以分解成四个峰, 分别位于 397.5, $399.1,400.1$ 和 $401.3 \mathrm{eV}$, 对应于吡啶 $\mathrm{N}$, 吡咯 $\mathrm{N}$, 石墨 $\mathrm{N}$ 和氧化 $\mathrm{N}^{[44]}$. $\mathrm{Fe} 、 \mathrm{Ni}$ 元素高分辨图谱(图 3b-c)表明, $\mathrm{Fe}$ 在表面呈现 $\mathrm{Fe}^{0}$ 和 $\mathrm{Fe}^{3+}$ 两种状态, $\mathrm{Ni}$ 呈现 $\mathrm{Ni}^{0}, \mathrm{Ni}^{2+}$ 两种 状态, 其中 $\mathrm{Fe}^{3+} 、 \mathrm{Ni}^{2+}$ 应为金属表面部分氧化所致. 结 合 XRD 分析表明, 所制备 $\mathrm{Fe}_{0.64} \mathrm{Ni}_{0.36} @ \mathrm{NC}$ 材料的碳层 内包裹了 $\mathrm{Fe}_{0.64} \mathrm{Ni}_{0.36}$ 合金以及少量单质 $\mathrm{Fe}$ 纳米颗粒, 金 属表面在空气中部分氧化, 同时元素 $\mathrm{N}$ 已成功掺杂到碳 层中.
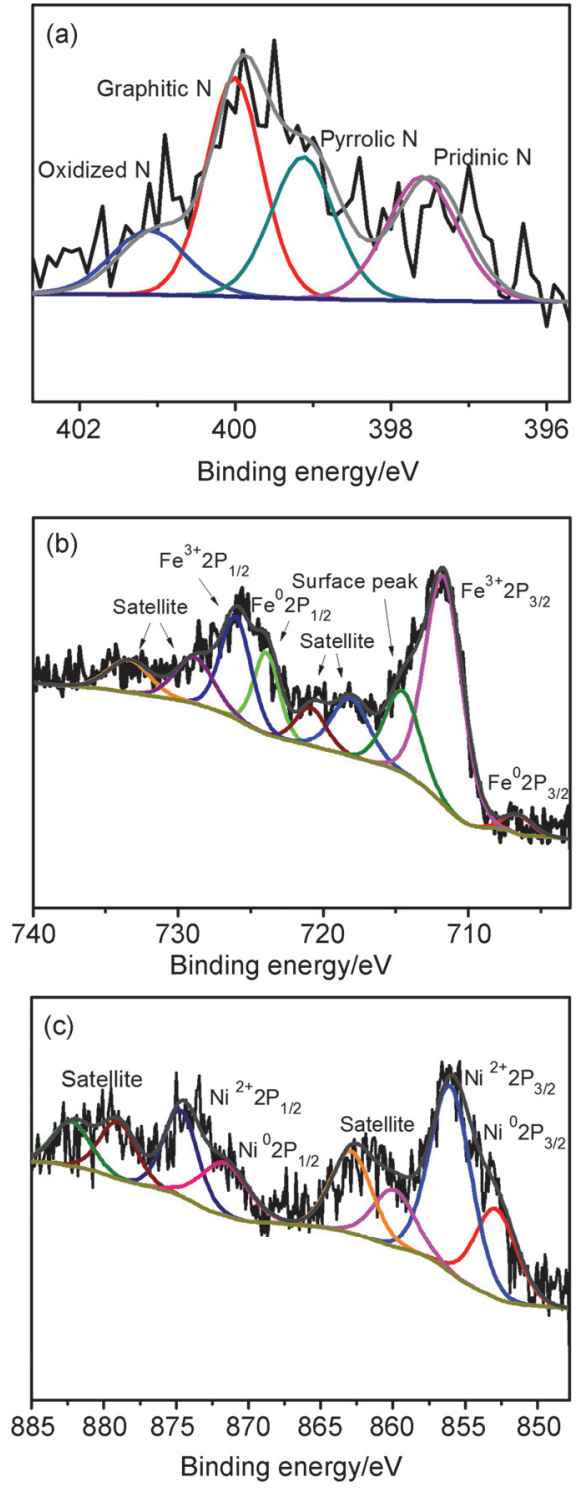

图 $3 \quad \mathrm{Fe}_{0.64} \mathrm{Ni}_{0.36} @ \mathrm{NC}$ 的 $\mathrm{N} 1 \mathrm{~s}(\mathrm{a}), \mathrm{Fe} 2 \mathrm{p}$ (b) 和 $\mathrm{Ni}$ (c)的高分辨 XPS 谱图 Figure 3 The high resolution XPS spectra of N 1s (a), Fe 2p (b) and Ni $2 \mathrm{p}$ (c) for $\mathrm{Fe}_{0.64} \mathrm{Ni}_{0.36} @ \mathrm{NC}$

图 4 的扫描电子显微镜(SEM)图片显示了所制备材 料的微观形貌结构. 图 $4 \mathrm{a}$ 是大小均匀的二氧化硅微球, 直径约为 $300 \mathrm{~nm}$. 加入 PDA 和 $\mathrm{Fe}^{3+}, \mathrm{Ni}^{2+}$ 充分反应后,
得到复合物 $\mathrm{Fe}^{3+}, \mathrm{Ni}^{2+}-\mathrm{PDA} @ \mathrm{SiO}_{2}$ (图 4b), 从图中可以 看出该复合物保留了二氧化硅球形形状且表面粗糙，说 明 PDA 已包覆在外层, 且没有出现明显的团聚现象. 图 $4 \mathrm{c}$ 是样品 $\mathrm{Fe}_{0.64} \mathrm{Ni}_{0.36} @ \mathrm{NC}$, 发现经过 $\mathrm{NaOH}$ 水热处理 24 $\mathrm{h}$ 后, $\mathrm{SiO}_{2}$ 小球刻蚀完全, 材料呈现中空结构. 为了了解 催化剂材料的结构、尺寸大小和晶格参数等信息，进一 步进行了透射电子显微镜(TEM)的表征. 从图 5 中可以 看出 $\mathrm{Fe}_{0.64} \mathrm{Ni}_{0.36} @ \mathrm{NC}$ 合金颗粒高度分散在碳氮化物层 中, 粒径约为 $20 \pm 2.2 \mathrm{~nm}$. 观察到间距为 $0.241 \mathrm{~nm}$ 和 $0.202 \mathrm{~nm}$ 的晶格, 分别归属于 $\mathrm{Fe}_{0.64} \mathrm{Ni}_{0.36}$ 合金的(111)晶 面 ${ }^{[45]}$ 和 $\mathrm{Fe}$ 单质的(110)晶面. 该结果进一步确认碳层内 包含 $\mathrm{Fe}_{0.64} \mathrm{Ni}_{0.36}$ 合金和单质 $\mathrm{Fe}$ ，与 XRD、XPS 分析结果 一致.
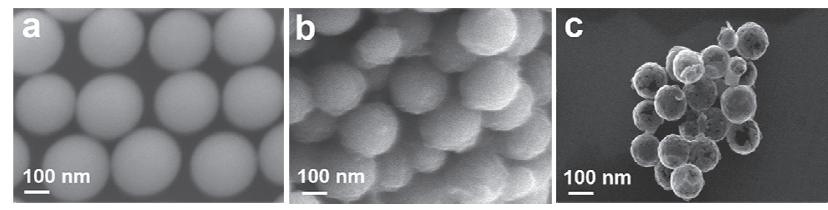

图 4 所制备催化剂的扫描电子显微镜图. (a) $\mathrm{SiO}_{2} ;$ (b) $\mathrm{Fe}^{3+}, \mathrm{Ni}^{2+}$. $\mathrm{PDA} @ \mathrm{SiO}_{2} ;$ (c) $\mathrm{Fe}_{0.64} \mathrm{Ni}_{0.36} @ \mathrm{NC}$

Figure 4 SEM images for the prepared products. (a) $\mathrm{SiO}_{2}$; (b) $\mathrm{Fe}^{3+}$, $\mathrm{Ni}^{2+}$-PDA@SiO $;$; (c) $\mathrm{Fe}_{0.64} \mathrm{Ni}_{0.36} @ \mathrm{NC}$

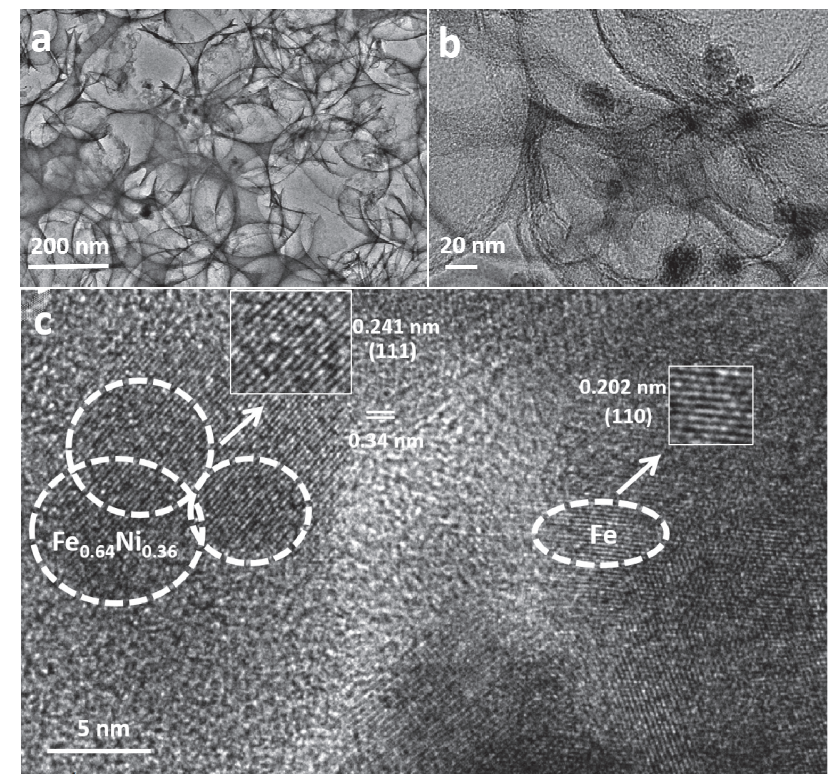

图 $5 \mathrm{Fe}_{0.64} \mathrm{Ni}_{0.36} @ \mathrm{NC}$ 的透射电子显微镜图

Figure 5 TEM images for the $\mathrm{Fe}_{0.64} \mathrm{Ni}_{0.36} @ \mathrm{NC}$

对所制备催化剂进行了氮气吸附脱附测试，表征其 孔道特征. 如图 6a 所示, 可以看出四种催化剂的等温吸 附/脱附均属于 IV 型吸脱附等温线, 在超低压力区具有 明显吸附, 在中压区 $p / p_{0}=0.5 \sim 0.8$ 具有明显的回滞环, 表明材料包含丰富的微孔和介孔. $\mathrm{Fe}_{0.64} \mathrm{Ni}_{0.36} @ \mathrm{NC}$ 催化 剂的比表面积是 $386.4 \mathrm{~m}^{2} \cdot \mathrm{g}^{-1}$, 孔径尺寸集中在 $7.0 \mathrm{~nm}$ 以下(图 6b), 结合 $\mathrm{SiO}_{2}$ 模板制造的 $300 \mathrm{~nm}$ 大孔, 所制备 材料应为包含微孔一介孔-大孔的多级孔结构. 


\section{2 电化学性能表征}

所制得催化剂在 $1.0 \mathrm{~mol} / \mathrm{L} \mathrm{KOH}$ 电解液中进行电化 学性能测试表征(三电极体系), 相应的结果如图 7 所示.

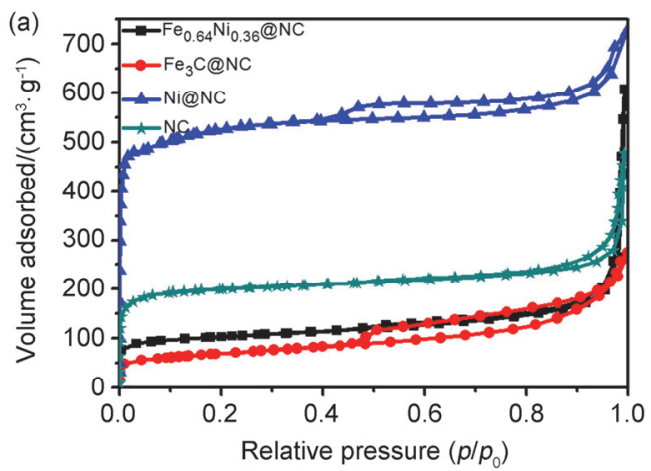

相比于 $\mathrm{Fe}_{3} \mathrm{C} @ \mathrm{NC} 、 \mathrm{Ni} @ \mathrm{NC}$ 和 $\mathrm{NC}, \mathrm{Fe}_{0.64} \mathrm{Ni}_{0.36} @ \mathrm{NC}$ 表现 出更优异的 OER 活性. 从图 7a 析氧极化曲线测试结果 对比图可以看出, $\mathrm{Fe}_{0.64} \mathrm{Ni}_{0.36} @ \mathrm{NC}$ 只需要 286 和 $333 \mathrm{mV}$

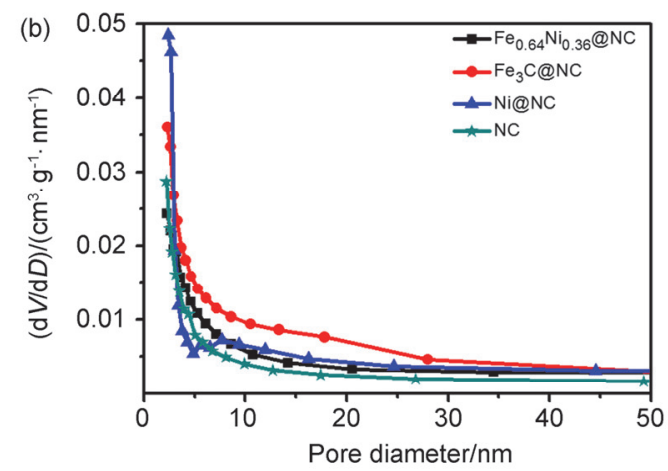

图 $6 \mathrm{Fe}_{0.64} \mathrm{Ni}_{0.36} @ \mathrm{NC}, \mathrm{Fe}_{3} \mathrm{C} @ \mathrm{NC}, \mathrm{Ni} @ \mathrm{NC}$ 和 $\mathrm{NC}$ 催化剂的 $\mathrm{N}_{2}$ 吸附/脱附等温线(a)和孔尺寸分布曲线(b)

Figure $6 \quad \mathrm{~N}_{2}$ adsorption-desorption isotherm (a) and pore size distribution plots (b) for $\mathrm{Fe}_{0.64} \mathrm{Ni}_{0.36} @ \mathrm{NC}, \mathrm{Fe}_{3} \mathrm{C} @ \mathrm{NC}, \mathrm{Ni} @ \mathrm{NC}$ and $\mathrm{NC}$ products
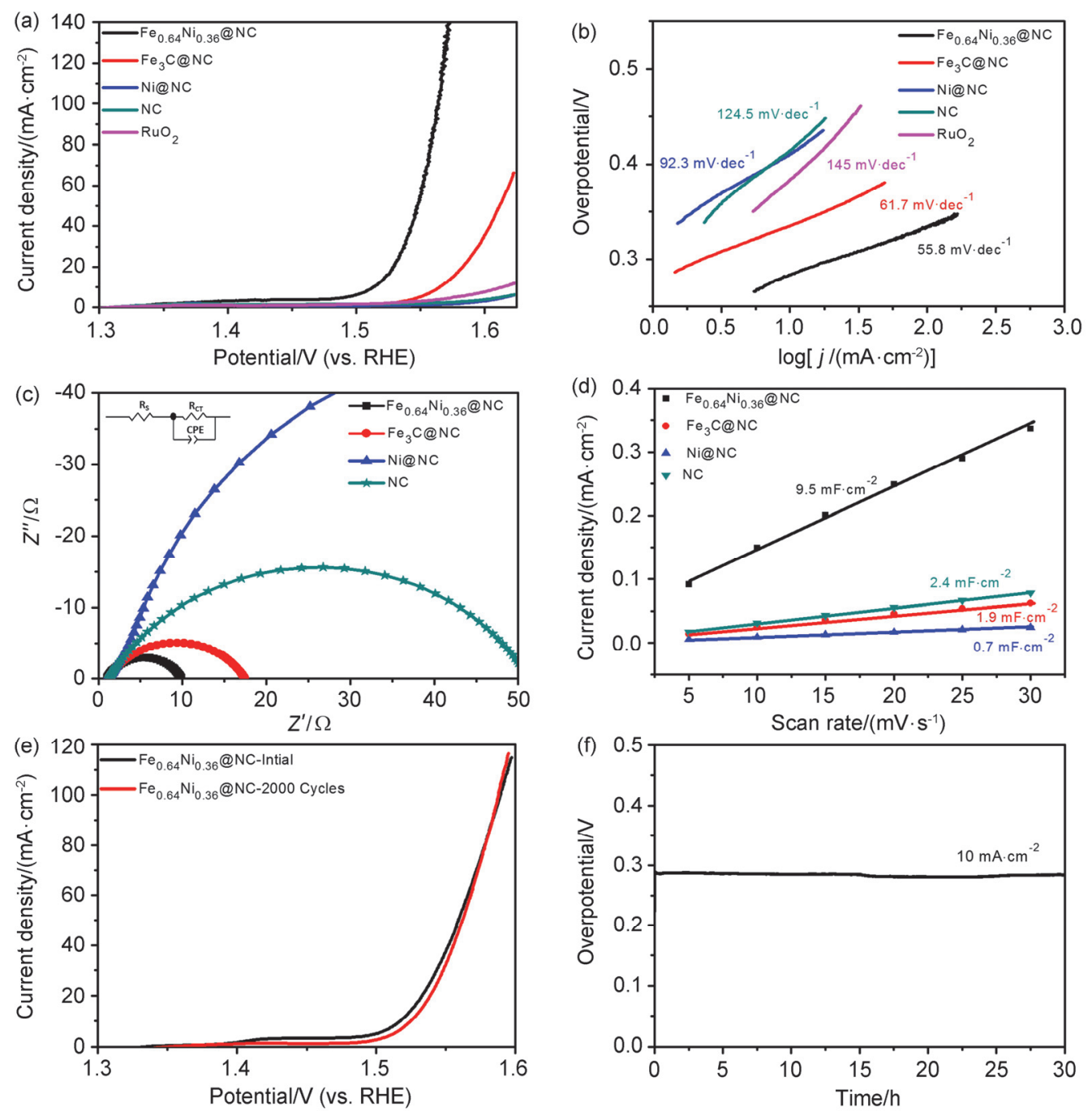

图 $7 \mathrm{Fe}_{0.64} \mathrm{Ni}_{0.36} @ \mathrm{NC}, \mathrm{Fe}_{3} \mathrm{C} @ \mathrm{NC}, \mathrm{Ni} @ \mathrm{NC}$ 和 $\mathrm{NC}$ 催化剂电化学析氧性能测试. (a)极化曲线; (b)Tafel 斜率; (c)电化学阻抗谱( (EIS); 尼奎斯特曲线的 等效电路(插图), (d)通过绘制 $1.0 \mathrm{~mol} / \mathrm{L} \mathrm{KOH}$ 中的电流密度变化 $\left(\Delta j=\left(j_{\mathrm{a}}-j_{\mathrm{c}}\right) / 2\right)$ 来估算 $C_{\mathrm{dl}} ;(\mathrm{e}, \mathrm{f}) \mathrm{Fe}_{0.64} \mathrm{Ni}_{0.36} @ \mathrm{NC}$ 催化剂的耐久性测试

Figure 7 The OER performance of the obtained catalysts: (a) polarization curves (with $i R$ correction); (b) Tafel slopes for the prepared materials (measured at a scan rate of $5.0 \mathrm{mV} \cdot \mathrm{s}^{-1}$ in $\mathrm{O}_{2}$-saturated $1.0 \mathrm{~mol} / \mathrm{L} \mathrm{KOH}$ solution); (c) Nyquist plots of the prepared catalysts; The inset shows the electrical equivalent circuit used to model the catalyst systems (inset); (d) An estimation of $C_{\mathrm{dl}}$ from plotting the current density variation $\left(\Delta j=\left(j_{\mathrm{a}}-j_{\mathrm{c}}\right) / 2\right)$ in $1.0 \mathrm{~mol} / \mathrm{L}$ $\mathrm{KOH} ;(\mathrm{e}, \mathrm{f})$ Durability test of $\mathrm{Fe}_{0.64} \mathrm{Ni}_{0.36} @ \mathrm{NC}$ catalyst 
的过电位就可以达到 10 和 $100 \mathrm{~mA} \cdot \mathrm{cm}^{-2}$ 的电流密度, 显著优于贵金属 $\mathrm{RuO}_{2}$ 催化剂在电流密度 $10 \mathrm{~mA} \cdot \mathrm{cm}^{-2}$ 时需要的 $380 \mathrm{mV}$ 的过电位 ${ }^{[46,47]}$, 也明显优于 $\mathrm{Fe}_{3} \mathrm{C} @ \mathrm{NC} 、 \mathrm{Ni} @ \mathrm{NC} 、 \mathrm{NC}$ 在 $10 \mathrm{~mA} \cdot \mathrm{cm}^{-2}$ 的电流密度时 的过电位. 另外, Tafel 斜率是评估 OER 动力学的重要指 标 ${ }^{[48]}$. 根据 LSV 曲线和 Tafel 斜率计算方程 $(\eta=$ $b \cdot \log \left(j / j_{0}\right)$, 其中 $\eta, j$ 和 $b$ 分别是过电位、电流密度和 Tafel 斜率), 可以得到如图 $7 \mathrm{~b}$ 所示的 Tafel 曲线. 显示样品 $\mathrm{Fe}_{0.64} \mathrm{Ni}_{0.36} @ \mathrm{NC}, \mathrm{Fe}_{3} \mathrm{C} @ \mathrm{NC}, \mathrm{Ni} @ \mathrm{NC}, \mathrm{NC}$ 的 Tafel 斜率分 别是 $55.8 \mathrm{mV} \cdot \mathrm{dec}^{-1}, 61.7 \mathrm{mV} \cdot \mathrm{dec}^{-1}, 92.3 \mathrm{mV} \cdot \mathrm{dec}^{-1}$ 和 $124.5 \mathrm{mV} \cdot \mathrm{dec}^{-1}$, 其中 $\mathrm{Fe}_{0.64} \mathrm{Ni}_{0.36} @ \mathrm{NC}$ 的斜率最小, 表 示其析氧极化随电流密度增大的速度最慢, OER 动力学 过程最快.

电化学阻抗谱(EIS)测试用来研究催化剂的电极反 应动力学 ${ }^{[49]}$. 在 $100 \mathrm{kHz}$ 至 $0.05 \mathrm{~Hz}$ 的频率范围内(图 $7 \mathrm{c})$, 所有样品中 $\mathrm{Fe}_{0.64} \mathrm{Ni}_{0.36} @ \mathrm{NC}$ 的电荷转移阻抗(8.79 $\Omega$ )最小, 动力学过程最快, 与 Tafel 数据结果一致. 进一 步测试材料在不同扫速下的 CV 曲线(图 S1), 并计算出 电化学活性表面积(electrochemically active surface area, $\mathrm{ECSA}$ ). 如图 7d 所示, $\mathrm{Fe}_{0.64} \mathrm{Ni}_{0.36} @ \mathrm{NC}$ 的 ECSA 为 9.5 $\mathrm{mF} \bullet \mathrm{cm}^{-2}$, 明显高于 $\mathrm{Fe}_{3} \mathrm{C} @ \mathrm{NC}\left(1.9 \mathrm{mF} \bullet \mathrm{cm}{ }^{-2}\right)$, $\mathrm{Ni} @ \mathrm{NC}\left(2.4 \mathrm{mF} \cdot \mathrm{cm}^{-2}\right)$ 和 $\mathrm{NC}\left(0.7 \mathrm{mF} \cdot \mathrm{cm}^{-2}\right)$. 最后测试了 $\mathrm{Fe}_{0.64} \mathrm{Ni}_{0.36} @ \mathrm{NC}$ 催化剂的稳定性, 在 1.3 1.6 V 电位下 连续扫描 2000 圈以后, 催化活性无明显衰减, 过电位仅 有少量增加(图 7e). 在电流密度 $10 \mathrm{~mA} \cdot \mathrm{cm}^{-2}$ 下连续测 试 $30 \mathrm{~h}$ 后, 电极输出非常稳定 (图 7f). 因此, $\mathrm{Fe}_{0.64} \mathrm{Ni}_{0.36} @ \mathrm{NC}$ 催化剂具有良好的电化学稳定性. 此外 进一步探究了不同 $\mathrm{Fe} / \mathrm{Ni}$ 比例、不同多巴胺含量制备的 催化剂在 OER 中的性能, 结果如图 S2 所示, 其中 $\mathrm{Fe}_{0.64} \mathrm{Ni}_{0.36} @ \mathrm{NC}$ 性能最好, 体现了原料最优化配比.

上述测试结果表明, $\mathrm{Fe}_{0.64} \mathrm{Ni}_{0.36} @ \mathrm{NC}$ 催化剂具有优 异的 OER 性能, 这来源于催化剂多重微观结构优势: (1) 微孔-介孔-大孔的多级孔结构有利于传质及暴露活性位 点, 提高活性位利用率; (2) 嵌入的 $\mathrm{Fe}_{0.64} \mathrm{Ni}_{0.36}$ 合金及单 质 $\mathrm{Fe}$ 颗粒和 $\mathrm{NC}$ 层协同作用, 产生丰富活性位点; (3)金 属颗粒由石墨化 NC 壳层包裹, 电化学反应过程中能很 好地保持原始形态和催化活性, 进而提升了催化剂抗腐 蚀能力和耐久性.

\section{3 结论}

本文以尺寸约为 $300 \mathrm{~nm}$ 二氧化硅微球为模板, 多 巴胺为氮碳源, $\mathrm{Fe}^{3+}, \mathrm{Ni}^{2+}$ 为金属源, 通过原位吸附、聚 合、焙烧、 $\mathrm{NaOH}$ 刻蚀得到铁镍合金纳米颗粒镶嵌的多 级孔氮掺杂碳催化剂 $\left(\mathrm{Fe}_{0.64} \mathrm{Ni}_{0.36} @ \mathrm{NC}\right)$. 在析氧反应中, 所制备的 $\mathrm{Fe}_{0.64} \mathrm{Ni}_{0.36} @ \mathrm{NC}$ 催化剂相对于贵金属 $\mathrm{RuO}_{2}$, 表现出优异的催化活性, 达到 $10 \mathrm{~mA} \cdot \mathrm{cm}^{-2}$ 的电流密度 时所需过电位 $286 \mathrm{mV}$, 同时该材料在连续扫描 2000 圈 后仍然保持较稳定的电极输出, 表现了良好的稳定性.
嵌入的 $\mathrm{Fe}_{0.64} \mathrm{Ni}_{0.36}$ 合金及 $\mathrm{Fe}$ 颗粒同 $\mathrm{NC}$ 外层协同作用, 产生丰富活性位点, 同时 $\mathrm{NC}$ 层的包覆大大提升了催化 剂稳定性. 本项工作设计的非贵金属催化剂廉价、高效、 耐用，为过渡金属催化剂在析氧方面的应用开辟了新的 思路.

\section{4 实验部分}

\section{1 铁镍合金纳米颗粒镶嵌的多级孔氮掺杂碳催化剂 的制备}

\section{1 .1 二氧化硅微球的合成}

$\mathrm{SiO}_{2}$ 合成基于 Stöber 方法 ${ }^{[50]}$. 首先, 将 $4.0 \mathrm{~mL}$ 氨水 溶液(25 28 wt\%)、10.0 mL 蒸馏水和 $60.0 \mathrm{~mL}$ 乙醇混合, 并向其中滴加 $10.0 \mathrm{~mL}$ 正硅酸乙酯(TEOS). 将该反应溶 液在 $30{ }^{\circ} \mathrm{C}$ 下连续搅拌 $4.5 \mathrm{~h}$, 得到直径约为 $300 \mathrm{~nm}$ 二氧 化硅微球悬浮液. 该悬浮液无需洗涤, 直接用于后续合 成.

4.1.2 铁镍合金纳米颗粒镶嵌的多级孔氮掺杂碳催化 剂的制备

在所制备的分散有 $\mathrm{SiO}_{2}$ 微球的悬浮液中加入 $10.0 \mathrm{~g}$ 多巴胺作为碳氮源, 然后加入 $1.5 \mathrm{mmoL} \mathrm{FeCl}_{3} \cdot 6 \mathrm{H}_{2} \mathrm{O}$ 和 $1.5 \mathrm{mmoL} \mathrm{NiCl}{ }_{2} \cdot 6 \mathrm{H}_{2} \mathrm{O}$ 作为铁源和镍源. 该溶液在 $30{ }^{\circ} \mathrm{C}$ 下连续摚拌 $24 \mathrm{~h}$ 后, 离心干燥得到 $\mathrm{Fe}^{3+}, \mathrm{Ni}^{2+}$ $\mathrm{PDA} @ \mathrm{SiO}_{2}$ 复合物. 将该样品在 $\mathrm{N}_{2}$ 中以 $2{ }^{\circ} \mathrm{C} / \mathrm{min}$ 升至 $200{ }^{\circ} \mathrm{C}$ 保持 $2 \mathrm{~h}$, 再以 $5{ }^{\circ} \mathrm{C} / \mathrm{min}$ 上升到 $800{ }^{\circ} \mathrm{C}$ 并保持 2 $\mathrm{h}$, 最后用 $3.0 \mathrm{~mol} / \mathrm{L} \mathrm{NaOH}$ 溶液在 $80{ }^{\circ} \mathrm{C}$ 下水热 $24 \mathrm{~h}$ 刻 蚀除去 $\mathrm{SiO}_{2}$ 模板, 用去离子水洗至中性, 并在 $60{ }^{\circ} \mathrm{C}$ 下 真空干燥, 得到样品 $\mathrm{Fe}_{0.64} \mathrm{Ni}_{0.36} @ \mathrm{NC}$. 此外, 我们采用 相同方法, 改变 $\mathrm{Fe}^{3+} 、 \mathrm{Ni}^{2+}$ 比例以及多巴胺加入量制得 几种对比样品, 详细合成参数见支撑信息.

\section{2 材料表征}

采用 FEI Nova 400 型扫描电子显微镜(SEM), 在 20 $\mathrm{kV}$ 电压条件下对样品尺寸和形貌进行分析; 采用 Zeiss LIBRA 200 型透射电子显微镜(TEM)在加速电压 $200 \mathrm{kV}$ 下观测样品的结构、晶格参数; 使用岛津 XRD-6000 型 $\mathrm{X}$ 射线衍射 (XRD) 设备表征晶体结构; 使用 Micrometrics Tristar 2390 型设备在 $77 \mathrm{~K}$ 下测量 $\mathrm{N}_{2}$ 吸附/ 脱附等温线, 在测量之前, 将样品在 $180{ }^{\circ} \mathrm{C}$ 下真空脱气 $10 \mathrm{~h}$.

\section{3 电化学性能表征}

电化学数据采用三电极体系在电化学分析仪 (CHI660D， CH Instruments，中国上海)上进行测量，对 电极为石墨棒 (直径 $10 \mathrm{~mm}$ ), 参比电极为 $\mathrm{Hg} / \mathrm{HgO}$, 电解 质是 $1.0 \mathrm{~mol} / \mathrm{L} \mathrm{KOH}$ 水溶液, 保持电解液达到 $\mathrm{O}_{2}$ 饱和状 态, 并确保所有实验在 $1.23 \mathrm{~V}$ vs. RHE下达到 $\mathrm{O}_{2} / \mathrm{H}_{2} \mathrm{O}$ 平 衡. 在 $1.3 \sim 1.7 \mathrm{~V}$ 的电位下以 $5 \mathrm{mV} \cdot \mathrm{s}^{-1}$ 的扫描速率测试 得到线性扫描伏安曲线(LSV). 恒电位状态下、 $1.0 \mathrm{~mol} / \mathrm{L}$ 
$\mathrm{KOH}$ 水溶液中, 在 $100 \mathrm{kHz}$ 至 $0.05 \mathrm{~Hz}$ 频率范围内测量 电化学阻抗谱(EIS). 所有极化曲线均针对 $i R$ 补偿进行 校正，计算公式如下:

$$
E_{\text {corrected }}=E_{\text {uncorrected }}-i R_{\mathrm{s}}
$$

其中 $i$ 和 $R_{\mathrm{s}}$ 代表电流密度和溶液阻抗.

$$
\begin{aligned}
& \text { 过电位计算如下: } \\
& \eta=E(\mathrm{vs} . \mathrm{RHE})-1.23
\end{aligned}
$$

在 $1.23 \mathrm{~V}$ vs. RHE 下的 $\mathrm{O}_{2} / \mathrm{H}_{2} \mathrm{O}$ 平衡.

塔菲尔斜率根据塔菲尔方程进行计算:

$$
\eta=b \cdot \log \left(j / j_{0}\right)
$$

其中, $\eta, b, j$ 和 $j_{0}$ 分别代表过电位，塔菲尔斜率，电流密 度和交换电流密度.

加速老化测试(accelerated durability tests, ADT)采 用循环伏安法，在 $1.3 \sim 1.6 \mathrm{~V}$ 高电位范围内进行 2000 次循环扫描, 以评估所制备催化剂在析氧反应中的电化 学耐久性.

\section{References}

[1] Armaroli, N.; Balzani, V. Angew. Chem., Int. Ed. 2007, 46, 52.

[2] Ding, W.; Zhang, X.; Li, L.; Wei, Z. D. J. Electrochem. 2014, 20, 426 (in Chinese). (丁炜, 张雪, 李莉, 魏子栋, 电化学, 2014, 20, 426.)

[3] Peng, L. S.; Wei, Z. D. Chinese J. Catal. 2018, 39, 1575 (in Chinese). (彭立山, 魏子栋, 催化学报, 2018, 39, 1575.)

[4] Villa, I.; Villa, C.; Monguzzi, A.; Babin, V.; Tervoort, E.; Nikl, M.; Niederberger, M.; Torrente, Y.; Vedda, A.; Lauria, A. Nanoscale 2018, 10, 7933 .

[5] Zhang, C.; Zhang, M. R.; Shi, H. Y.; Wang, J. P.; Niu, J. Y. Chem. Commun. 2018, 54, 5458.

[6] Guo, Y.; Yao, Y.; Li, H.; He, L. L.; Yang, Z. Z.; Zhao, D. X. Acta Chim. Sinica 2017, 75, 903 (in Chinese). (郭宇, 姚宇, 李慧, 赫兰 兰, 杨忠志, 赵东霞, 化学学报, 2017, 75, 903.)

[7] Zuo, L. X.; Jiang, L.; Zhu, J. J. Chin. J. Chem. 2017, 35, 969.

[8] Zou, X.; Zhang, Y. Chem. Soc. Rev. 2015, 44, 5148.

[9] Wang, S. L.; Wang, L. P.; Zhang, Z. H. Acta Phys.-Chim. Sin. 2013, 29, 981 (in Chinese). (王森林, 王丽品, 张振洪, 物理化 学学报, 2013, 29, 981.)

[10] Wang, J.; Wei, Z. D. Acta Phys.-Chim. Sin. 2017, 33, 886 (in Chinese). (王俊, 魏子栋, 物理化学学报, 2017, 33, 886.)

[11] Peng, L. S.; Wei, Z. D. Prog. Chem. 2018, 30, 14 (in Chinese). (彭 立山，魏子栋，化学进展, 2018, 30, 14.)

[12] Luo, J. L. Acta Phys.-Chim. Sin. 2018, 34, 7 (in Chinese). (骆静利, 物理化学学报, 2018, 34, 7.)

[13] Zhang, Y.; Shimoda, K.; Miyaoka, H. Int. J. Hydrogen Energy 2010, 35,12405 .

[14] Hinnemann, B.; Moses, P. G.; Bonde, J.; Jorgensen, K. P.; Nielsen, J. H. J. Am. Chem. Soc. 2005, 127, 5308.

[15] Lee, Y.; Jin, S.; May, K. J.; Perry, E. E.; Yang, S. H. J. Phys. Chem. Lett. 2012, 3, 399.

[16] Over, H. Chem. Rev. 2012, 43, 3356.

[17] Liu, T. T.; Xie, L. S.; Yang, J. H.; Kong, R. M.; Sun, X. P.; Chen, L. ChemElectroChem 2017, 4, 1840

[18] Fang, Y. H.; Liu, Z. P. J. Am. Chem. Soc. 2010, 132, 18214.

[19] Fang, Z.; Peng, L.; Zhu, Y.; Yan, C.; Wang, S.; Kalyani, P.; Wu, X.;
Yu, G. ACS Nano 2017, 11, 9550.

[20] Feng, J. X.; Xu, H.; Dong, Y. T.; Ye, S. H.; Tong, Y. X.; Li, G. R. Angew. Chem., Int. Ed. 2016, 128, 3758.

[21] Ji, Y. Y.; Li, Y.; Ren, X.; Cui, G. W.; Xiong, X. L.; Sun, X. P. ACS Sustain. Chem. Eng. 2018, 6, 9555.

[22] Yang, J.; Zhu, G.; Liu, Y.; Xia, J.; Ji, Z.; Shen, X. Adv. Funct. Mater. 2016, 26, 4712.

[23] Gao, M.; Sheng, W.; Zhuang, Z.; Fang, Q.; Gu, S.; Jiang, J.; Yan, Y. J. Am. Chem. Soc. 2014, 136, 7077 .

[24] Chuan, T. Y.; Chun, Z. H. Chem. Commun. 2016, 52, 11591.

[25] Hoare, J. P.; Schuldiner, S. J. Phys. Chem. 2002, 62, 229

[26] Tang, T.; Jiang, W. J.; Niu, S.; Liu, N.; Luo, H.; Chen, Y. Y.; Jin, S. F.; Gao, F.; Wan, L. J. J. Am. Chem. Soc. 2017, 139, 8320.

[27] Li, F. L.; Shao, Q.; Huang, X.; Lang, J. P. Angew. Chem., Int. Ed. 2018, 57,1888 .

[28] Chemelewski, W. D.; Rosenstock, J. R.; Mullins, C. B. J. Mater. Chem. 2014, 2, 14957.

[29] He, Y. H.; Xu, J. M.; Wang, N. Chem. Eng. Prog. 2016, 35, 2057 (in Chinese). (何杨华, 徐金铭, 王楠, 化工进展, 2016, 35, 2057.)

[30] Landon, J.; Demeter, E.; İnoğlu, N.; Keturakis, C.; Wachs, I. E.; Frenkel, A. I. ACS Catal. 2012, 2, 1793.

[31] Gong, M.; Li, Y.; Wang, H.; Liang, Y.; Wu, J. Z.; Zhou, J.; Wang, J.; Regier, T.; Wei, F. J. Am. Chem. Soc. 2013, 135, 8452.

[32] Trotochaud, L.; Young, S. L.; Ranney, J. K. J. Am. Chem. Soc. 2014, 136, 6744.

[33] Smith, A. M.; Trotochaud, L.; Burke, M. S.; Boettcher, S. W. Chem. Commun. 2015, 51, 5261.

[34] Yang, Y.; Zhuang, L.; Lin, R.; Li, M.; Xu, X.; Rufford, T. E.; Zhu, Z. J. Power Sources 2017, 349, 68 .

[35] Liang, Y.; Liu, Q.; Asiri, A. M.; Sun, X.; He, Y. Int. J. Hydrogen Energy 2015, 40, 13258

[36] Zhao, Y.; Chen, S.; Sun, B.; Su, D.; Huang, X.; Liu, H.; Yan, Y.; Sun, K.; Wang, G. Sci. Rep. 2015, 5, 7629.

[37] Ma, T. Y.; Dai, S.; Jaroniec, M.; Qiao, S. Z. J. Am. Chem. Soc. 2014, 136,13925

[38] Yang, Y.; Lin, Z.; Gao, S.; Su, J.; Lun, Z.; Xia, G. ACS Catal. 2017 7, 469 .

[39] Tavakkoli, M.; Kallio, T.; Reynaud, O.; Nasibulin, A. G.; Johans, C.; Sainio, J.; Jiang, H.; Kauppinen, E. I.; Laasonen, K. Angew. Chem., Int. Ed. 2015, 54, 4535.

[40] Tao, Z.; Wang, T.; Wang, X.; Zheng, J.; Li, X. ACS Appl. Mater. Interfaces 2016, 8,35390

[41] Liu, Z.; Sun, F.; Gu, L.; Chen, G.; Shang, T.; Liu, J.; Le, Z.; Li, X.; Wu, H. B.; Lu, Y. Adv. Energy Mater. 2017, 7, 1701154.

[42] Guo, Y.; Liu, Y.; Qi, J. J.; Li, H.; He, L. L. Acta Chim. Sinica 2017, 75,914 (in Chinese). (郭宇, 刘瑜, 戚娟娟, 李慧, 赫兰兰, 化学 学报, 2017, 75,914 .)

[43] Bao, J. Z.; Wang, S. L. Acta Phys.-Chim. Sin. 2011, 27, 2849 (in Chinese). (鲍晋珍, 王森林, 物理化学学报, 2011, 27, 2849.)

[44] Yang, Q. Q.; Liu, L.; Xiao, L.; Wang, M. J.; Li, J.; Wei, Z. D. J. Mater. Chem. A 2018, 6, 14752.

[45] Ma, Y. D.; Dai, X. P.; Liu, M. Z.; Zhang, X. ACS Appl. Mater. Interfaces 2016, 8, 34396.

[46] Wu, Y.; Chen, M.; Han, Y.; Luo, H.; Su, X.; Zhang, M. T.; Lin, X.; Sun, J.; Wang, L.; Deng, L.; Zhang, W.; Cao, R. Angew. Chem., Int Ed. 2015, 54, 4870 .

[47] Zhang, B.; Xiao, C.; Xie, S.; Liang, J.; Chen, X.; Tang, Y. Chem. Mater. 2016, 28, 6934.

[48] Han, X.; Yu, C.; Zhou, S.; Zhao, C.; Huang, H.; Yang, J.; Liu, Z.; Zhao, J.; Qiu, J. Adv. Energy Mater. 2017, 7, 1602148.

[49] Wu, G.; Li, N.; Dai, C. S.; Zhou, D. R. Chinese J. Catal. 2004, 25, 319 (in Chinese). (武刚, 李宁, 戴长松, 周德瑞, 催化学报, 2004, 25, 319.)

[50] Stöber, W.; Fink, A.; Bohn, E. J. Colloid Interface Sci. 1968, 26, 62.

(Cheng, B.) 